\title{
Correction to: Environmental Justice 2017;10(3): 72-80. DOI: 10.1089/env.2016.0043
}

$\mathbf{I}^{\mathrm{N}}$

N THE JUNE 2017 issue of Environmental Justice (vol. 10, no. 3, pp. 72-80), there are errors in the Abstract and in the Acknowledgments of the article entitled "Urban Water Insecurity: Case Study of Homelessness in Phoenix, Arizona" by DeMeyers, Warpinski, and Wutich.

In the Abstract on page 72 of the article, the first two sentences should be changed. The incorrect first two sentences of the abstract:

\begin{abstract}
"In this research project we engage with the misconception that all people in the United States enjoy water security by examining the case of people experiencing homelessness in the city of Phoenix Arizona, in the southwestern United States. People who experience homelessness are disproportionately at risk of dehydration and heat-related illness as they spend significantly more time outdoors, and many have limited access to an adequate quantity of acceptable quality water.'
\end{abstract}

should be corrected to read:

"People who experience homelessness are disproportionately at risk of dehydration and heat-related illness as they spend significantly more time outdoors, and many have limited access to an adequate quantity of acceptable quality water. Insufficient access to water can occur in wealth, highly developed countries that have well-developed water infrastructure and water treatment systems, such as the United States."

In the Acknowledgments on page 80 of the article, an additional source of funding should be added. The incorrect Acknowledgments:

"This material is based on work supported by the National Science Foundation under Grant Nos. DEB-1637590 and DEB-1026865, Central Arizona-Phoenix Long-Term Ecological Research (CAP LTER).'

should be corrected to read:

“This material is based on work supported by the National Science Foundation under Grant Nos. DEB-1637590 and DEB-1026865, Central Arizona-Phoenix Long-Term Ecological Research (CAP LTER); and Grant No. SES0951366, DMUU: Decision Center for a Desert City II: Actors, Governance, and Institutions."

The online version of the article has been corrected to reflect the changes.

The authors wish to apologize for the errors. 